This item was submitted to Loughborough's Research Repository by the author.

Items in Figshare are protected by copyright, with all rights reserved, unless otherwise indicated.

\title{
Channel shortening filter design based on polynomial methods
}

PLEASE CITE THE PUBLISHED VERSION

PUBLISHER

(C) IEEE

VERSION

VoR (Version of Record)

LICENCE

CC BY-NC-ND 4.0

REPOSITORY RECORD

Toker, Cenk, Jonathon Chambers, and Buyurman Baykal. 2019. "Channel Shortening Filter Design Based on Polynomial Methods". figshare. https://hdl.handle.net/2134/5911. 
This item was submitted to Loughborough's Institutional Repository (https://dspace.lboro.ac.uk/) by the author and is made available under the following Creative Commons Licence conditions.

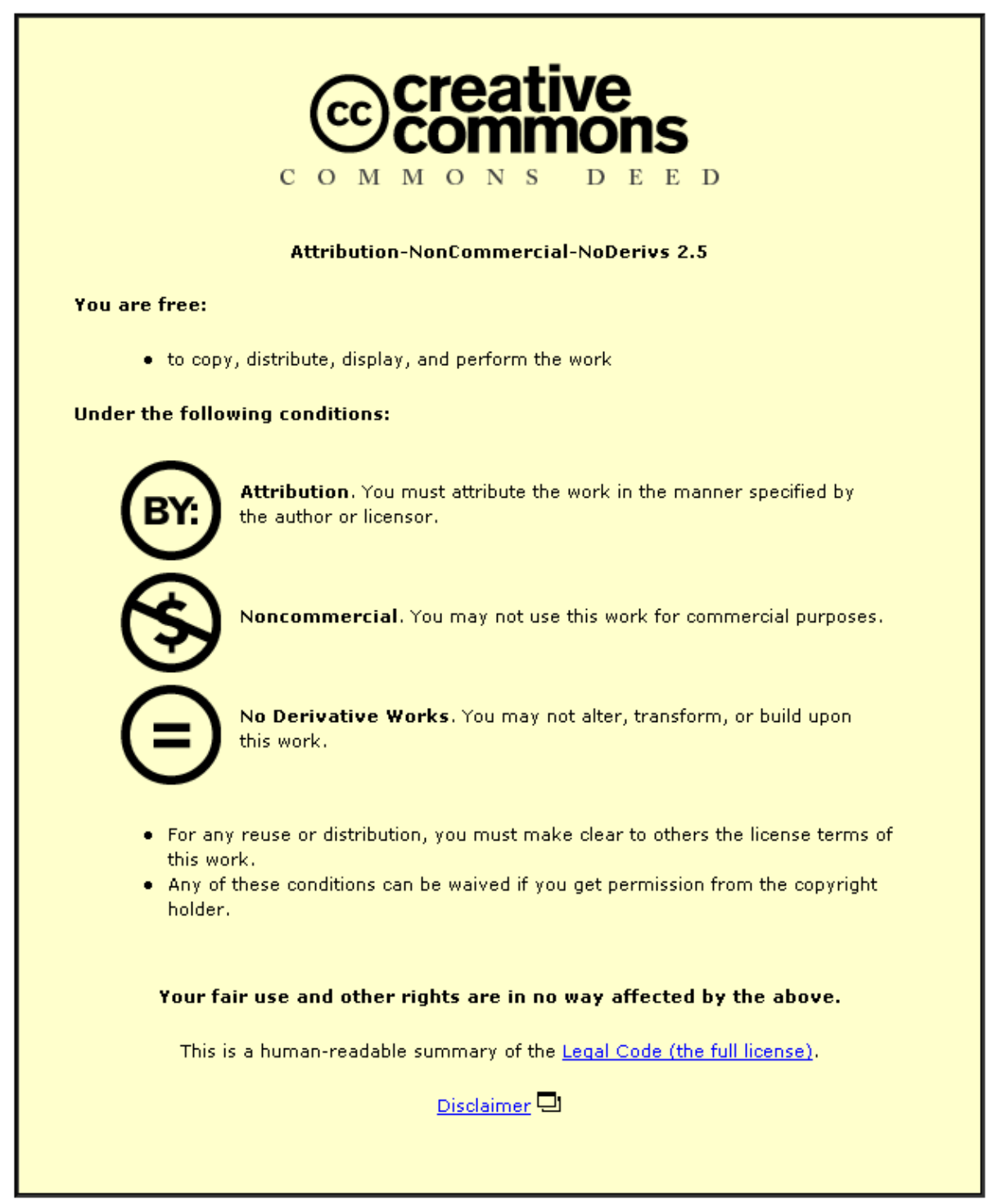

For the full text of this licence, please go to: http://creativecommons.org/licenses/by-nc-nd/2.5/ 


\section{Channel Shortening Filter Design Based on Polynomial Methods}

\author{
Cenk Toker \\ and Sangarapillai Lambotharan \\ CDSPR, King's College London, \\ Strand Campus, WC2R 2LS, London, U.K.
}

\author{
Jonathon A. Chambers \\ Cardiff School of Engineering, \\ Cardiff University, Queen`s Buildings, \\ Cardiff, CF24 OYF, Wales, UK
}

\author{
Buyurman Baykal \\ Dept. Electrical and Electronics Eng., \\ Middle East Technical University, \\ 06530, Ankara, Turkey
}

\begin{abstract}
Intersymbol interference (ISI) is a major cause of performance degradation for both wireless and wireline communication systems. It can be mitigated by several different methods including equalization and multicarrier modulation, but the complexity and efficiency of all methods would depend on the length of the ISI channel. In this paper, we propose a general framework for channel shortening where we considered the channel as a rational transfer function (having infinite impulse response (IIR)), and the source and the noise as autoregressive moving average (ARMA) processes. The aim is to shorten the channel using an IIR filter to a desirable length, so that computationally efficient post processing techniques can be applied to the resulting signal. The use of an IIR filter provides more degrees of freedom for channel shortening as compared to an FIR filter.
\end{abstract}

\section{A. Introduction}

Intersymbol interference (ISI) caused by frequency selectivity of the channel is one of the major factors which degrades the performance of both wireless and wireline communication systems. A great amount of research has been conducted to combat ISI with channel equalization. It is well known that a maximum likelihood sequence estimator (MLSE) has a performance superior to a linear equalizer but its complexity increases exponentially with the length of the channel. In the literature, several attempts have been made to reduce the complexity while retaining a performance close to the full complexity counterpart, these include reduced state MLSE [7] and channel shortening methods [8],[11].

Another method is based upon multicarrier modulation techniques which transform the frequency selective fading channel into independent parallel flat fading subchannels. A cyclic prefix is added to the transmission packet to eliminate interblock interference (IBI). The length of the cyclic prefix must be greater than or equal to the order of the ISI channel. Therefore the transmission efficiency is limited by the length of the channel. Again, to reduce the channel length, channel shortening equalizers can be employed [10].

In the literature, there have been several attempts to design good channel shortening filters. For the MLSE equalizer the main approach is the minimum mean square error method under a unit-tap or a unit energy constraint, [4], [8]. For multicarrier modulation systems besides the minimum mean square error (MMSE) solution several other methods have also been proposed such as the geometric signal to noise ratio
(SNR) and maximum bit rate (MBR) solutions [6], [5], [10] In this paper, however, we will focus on the MMSE criterion applied to an IIR based channel shortening filter based on a polynomial approach.

It is well known that the zeros of a system can be perfectly compensated for the poles of an IIR equalizer. If a zero of the channel is very close to the unit circle, the IIR filter is required to place a pole at that location for a zero forcing solution. For the MMSE solution, as it takes noise into account, it does not exactly cancel the zero but places a pole very close to the position of the zero. Both solutions, however, result in a very long impulse response. An FIR filter can also be employed to approximate this impulse response, but if the length of the filter is not adequate, the resulting error could be much higher than that of using an IIR filter.

A known problem associated with an IIR filter is its noise amplification due to infinite length of the impulse response, but as it will be demonstrated a superior channel shortening performance could compensate for the noise amplification when the signal-to-interference plus noise ratio is considered. Another important issue which needs special treatment in IIR filter design is the stability problem. This problem is carefully addressed in the design by choosing stable poles through spectral factorization.

To our best knowledge, there has been only one attempt to tackle channel shortening filter design using IIR filters. In [5], the channel shortening filter is first designed using a very long FIR filter, and then this long FIR filter is approximated to an IIR filter, [3].

In this paper we propose a frequency domain approach based on polynomial equations [1], [2] to design IIR channel shortening filters directly under the criterion of MMSE. We consider a general framework by allowing the channel, noise and transmitted signal models to be auto-regressive moving average (ARMA) filters, but special cases such as FIR channels, white signal and noise sources can be readily obtained from the general approach.

For a polynomial of length $n P+1$,

$$
P\left(q^{-1}\right)=p_{0}+p_{1} q^{-1}+\ldots+p_{n P} q^{-n P}
$$

the conjugate polynomial is defined as

$$
P_{*}(q)=p_{0}^{*}+p_{1}^{*} q+\ldots+p_{n P}^{*} q^{n P} .
$$




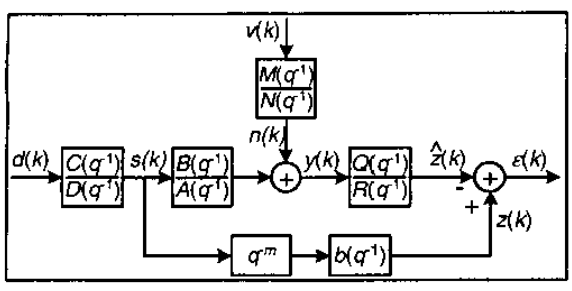

Fig. 1. The general framework for the channel shortening method, when the source and noise processes are ARMA and the channel has a rational transfer function. The channel is assumed to be shortened to a finite length FIR filter.

where $q^{-1}$ is the backward shift operator, i.e. $q^{-1} x(k)=x(k-$ $1)$, and $(\cdot)^{*}$ is the complex conjugate operator. A polynomial $P\left(q^{-1}\right)$ is called stable if all of its zeros are located inside the unit circle, and causal when the polynomial contains only the term $q^{-1}$, similarly anticausal if it is composed of positive power of $q$.

\section{Problem Statement}

Consider the structure in Figure 1 depicting the channel, target impulse response and the shortening filter together with the input data and noise models. Data source and noise processes, $s(k)$ and $n(k)$, are parametrized by ARMA models, $C\left(q^{-1}\right) / D\left(q^{-1}\right)$ and $M\left(q^{-1}\right) / N\left(q^{-1}\right)$ respectively. The channel is assumed to have a rational transfer function, $B\left(q^{-1}\right) / A\left(q^{-1}\right)$. The ARMA source model for the data could model certain preprocessing techniques applied to the signal before transmission. All polynomials except $B\left(q^{-1}\right)$ are monic and furthermore the polynomials $D\left(q^{-1}\right), A\left(q^{-1}\right)$ and $N\left(q^{-1}\right)$ are assumed to be stable. The signals $d(k)$ and $v(k)$ are assumed to be mutually independent, stationary, white and zero mean, with variances $\lambda_{d}$ and $\lambda_{v}$, respectively. The channel shortening filter is modelled by a causal and stable rational transfer function, $Q\left(q^{-1}\right) / R\left(q^{-1}\right)$. The polynomials $Q\left(q^{-1}\right)$ and $R\left(q^{-1}\right)$ are assumed to be coprime, otherwise the common terms will cancel each other. The channel shortening filter is designed to shorten the channel to a finite length window with a causal target impulse response (TIR) polynomial, $b\left(q^{-1}\right)=b_{0}+b_{1} q^{-1}+\ldots+b_{n b} q^{-n b}$, where $n b$ is the order of the TIR. The delay parameter $m$ is adjusted to maximize the performance.

The input to the channel shortening filter is

$$
y(k)=\frac{B\left(q^{-1}\right)}{A\left(q^{-1}\right)} \frac{C\left(q^{-1}\right)}{D\left(q^{-1}\right)} d(k)+\frac{M\left(q^{-1}\right)}{N\left(q^{-1}\right)} v(k)
$$

This signal can be written in the innovations form as

$$
y(k)=\frac{\beta\left(q^{-1}\right)}{A\left(q^{-1}\right) D\left(q^{-1}\right) N\left(q^{-1}\right)} \sqrt{\lambda_{\eta}} \eta(k)
$$

where $\beta\left(q^{-1}\right)=1+\beta_{1} q^{-1}+\ldots+\beta_{n \beta} q^{-n \beta}$ is the monic, causal and stable spectral factor polynomial, $\eta(k)$ is the zero mean and white innovations process with variance unity, and $\lambda_{\eta}$ is a gain factor. Equating the power spectral densities of both definitions of $y(k)$ in (3) and (4), we obtain

$$
\begin{gathered}
\frac{r \beta \beta_{*}}{A A_{*} D D_{*} N N_{*}}=\frac{B B_{*} C C_{*}}{A A_{*} D D_{*}}+\rho \frac{M M_{*}}{N N_{*}} \\
r \beta \beta_{*}=B B_{*} C C_{*} N N_{*}+\rho A A_{*} D D_{*} M M_{*}
\end{gathered}
$$

where $r=\lambda_{\eta} / \lambda_{d}$, and $\rho=\lambda_{n} / \lambda_{d}$. It is clear that the order of $\beta\left(q^{-1}\right)$ is $n \beta=\max \{n B+n C+n N, n A+n D+n M\}$. Parentheses are dropped where convenient.

The aim is to design the shortening filter $Q\left(q^{-1}\right) / R\left(q^{-1}\right)$ and the TIR $b\left(q^{-1}\right)$ to reduce the length of the equalized channel to $n b+1$ taps while minimizing the difference between the equalized channel and the TIR in the MMSE sense. After shortening, most of the energy of the original channel will be compressed to the window between the $m^{\prime}$ th and $(m+n b)$ th taps.

\section{IIR ChANNEL SHORTENING FILTER}

The error signal which is a measure of the difference between the target impulse response and the shortened channel can be expressed as

$$
\begin{aligned}
\varepsilon(k) & =z(k)-\hat{z}(k) \\
& =\left[q^{-m} b-\frac{Q}{R} \frac{B}{A}\right] \frac{C}{D} d(k)-\frac{Q}{R} \frac{M}{N} v(k)
\end{aligned}
$$

The aim of the channel shortening filter is to minimize the mean square error (MSE) cost function, with respect to the shortening filter $Q\left(q^{-1}\right) / R\left(q^{-1}\right)$ and the TIR $b\left(q^{-1}\right)$,

$$
\begin{aligned}
& J= E\left\{\varepsilon(t) \varepsilon^{*}(t)\right\} \\
&=\frac{\lambda_{d}}{2 \pi j} \oint_{|z|=1} r\left[\frac{Q \beta}{R A D N}-\frac{z^{-m} b B_{*} C C_{*} N_{*}}{r \beta_{*} D}\right] \times \\
& \\
& {\left[\frac{Q_{*} \beta_{*}}{R_{*} A_{*} D_{*} N_{*}}-\frac{z^{m} b_{*} B C C_{*} N}{r \beta D_{*}}\right]+} \\
& \rho \frac{b b_{*} A A_{*} C C_{*} M M_{*}}{r \beta \beta_{*}} \frac{d z}{z}
\end{aligned}
$$

where we used Parseval's formula (the term $q^{-1}$ is replaced by $z^{-1}$ where appropriate) and completed the squares for the terms involving the shortening filter coefficients. It can be seen that the MSE depends on both the shortening filter and the TIR coefficients. The first step is to find the shortening filter solution as a function of the TIR, by minimizing the MSE. coefficients. Then back substituting this solution to the MSE in (9), we obtain the MSE depending only on the TIR coefficients which requires a second set of optimization.

We could observe that the first term in the first parenthesis in (9) is strictly causal, stable and contains the transfer function of the shortening filter, $Q / R$, while the second term is mixed. We can infer that the best that the shortening filter could do to minimize the MSE is to cancel the causal part of the second term in the first parenthesis in (9). The same reasoning also applies to the second parenthesis, but in this case the shortening filter cancels the anticausal terms. Decomposing the 
second term into its causal and anticausal factors, we could write

$$
\frac{z^{-m} b B_{*} C C_{*} N_{*}}{r \beta_{*} D}=\frac{Q_{1}}{D}+\frac{z L_{*}}{r \beta_{*}}
$$

where $Q_{1}$ and $z L_{*}$ are respectively strictly causal and anticausal polynomials satisfying the equality. The order of $Q_{1}$ is $n Q_{1}=\max \{n b+n C+m, n D-1\}$ and the order of $L_{*}$ is $n L=\max \{n B+n C+n N-m, n \beta\}-1$.

Equivalently, we can rewrite (10) as

$$
z^{-m} b B_{*} C C_{*} N_{*}=r \beta_{*} Q_{1}+z D L_{*}
$$

which is a Diophantine equation and there is an efficient method to solve this equation [9], [1]. The left hand side term is written in the vector form where each row corresponds to a time delay, and the right hand side can be written as the multiplication of a convolution matrix containing the coefficients $r \beta_{*}$ and $z D$, and a vector containing the coefficients of $Q_{1}$ and $L_{*}$. By solving this system of equations one can easily find $Q_{1}$ and $L_{*}$ depending on $b\left(q^{-1}\right)$.

Comparing (9) and (10) we conclude that $Q \beta / R A D N$ cancels $Q_{1} / D$, i.e.

$$
\begin{aligned}
& \frac{Q \beta}{R A D N}=\frac{Q_{1}}{D} \\
& \frac{Q}{R}=\frac{Q_{1} A N}{\beta}
\end{aligned}
$$

Here we should note some facts; the numerator directly cancels the stable and causal spectral factor of the filter input, and the numerator has factors cancelling the poles of the channel and noise processes together with a third term, $Q_{1}$. Actually the term $A N / \beta$ is the inverse of the rational transfer function in the innovations model, therefore the channel shortening filter first tries to whiten the input signal (since D is not included in the design branch (Figure 1) it is not included in this cancellation). With the selection of the shortening filter coefficients as in (13) the first parenthesis in (9) results in a strictly anticausal expression, $-z L_{*} / r \beta_{*}$, which is the best that a strictly causal and stable shortening filter can do. Since $Q_{1}$ and $z L_{*}$ are strictly causal and anticausal, respectively, they are mutually independent. Therefore both of them are functions of the TIR coefficients, $b\left(q^{-1}\right)$, but they do not depend on each other. The same result could also be obtained by utilizing the principle of orthogonality,

$$
=\frac{\lambda_{d}}{2 \pi j} \oint_{|=|=1}\left\{\frac{z^{-m} R b A B_{*} C C_{*} N N_{*}-r \beta \beta_{*} Q}{R A A_{*} D D_{*} N N_{*}}\right\} \frac{d z}{z}
$$

Cauchy's integral theorem states that the contour integration of a rational function yields zero if all of the poles of that function are located outside the contour. Therefore the numerator should cancel all the poles inside the unit circle to minimize the MSE, i.e.

$$
z^{-m} R b A B_{*} C C_{*} N N_{*}-r \beta \beta_{*} Q=z R A D N L_{*}
$$

In a polynomial equation with three terms, if a factor is common to two terms, it must also be a factor of the third one. Since $R$ is common to the first and the third terms, it must also be included in the second term. Since the only stable factor in the second term is $\beta$ (note that the polynomials $Q$ and $R$ are coprime hence only $\beta$ is considered), it follows that $R=\beta$. Using a similar reasoning we can determine that $Q=Q_{1} A N$.

Back substituting the solution in (13) into (9), we obtain

$$
J=\frac{\lambda_{d}}{2 \pi j} \oint_{|=|=1}\left\{\frac{L L_{*}+\rho b b_{*} A A_{*} C C_{*} M M_{*}}{r \beta \beta_{*}}\right\} \frac{d z}{z}
$$

Since $L_{*}(q)$ can be solved from (11) as a function of $b\left(q^{-1}\right)$, the only unknown in (17) is the TIR $b\left(q^{-1}\right)$. Cauchy's integral formula states that

$$
\frac{1}{2 \pi j} \oint_{|z|=1} \frac{k}{(z-a)^{m}} d z=\left\{\begin{array}{lr}
k, & |a|<1, m=1 \\
0, & \text { otherwise }
\end{array}\right.
$$

Therefore the only poles contributing to the integral in (17) are $z$ and the roots of $\beta\left(q^{-1}\right)$. After utilizing a partial fraction expansion to find the numerators of these $n \beta+1$ terms and summing them up, (17) can be expressed as a quadratic expression with the coefficients of $b\left(q^{-1}\right)$ as variables. In matrix notation

$$
J=\mathbf{b}^{H} \mathbf{R} \mathbf{b}
$$

where $\mathbf{b}=\left[\begin{array}{llll}b_{0} & b_{1} & \cdots & b_{n b}\end{array}\right]^{T}$, and the matrix $\mathbf{R}$ is positive semidefinite. A loose proof follows from the intuitive reasoning that the left hand side of (19) corresponds to a nonnegative quantity (power). The MSE can be minimized by choosing $\mathbf{b}$ as the eigenvector corresponding to the smallest eigenvalue of $\mathrm{R}$. Hence, the proposed method has a unit energy TIR which means the shortening filter forces the equalized channel to have unity gain.

The equalized channel after performing the channel shortening is

$$
\begin{aligned}
C e q & =\frac{B}{A} \frac{Q}{R} \\
& =\frac{Q_{1} B N}{\beta}
\end{aligned}
$$

\section{COMPARISON OF THE IIR FILTER WITH THE FIR FILTER}

In this section we will compare the proposed method with the FIR MMSE channel shortening approach, [4], under unit energy criterion (UEC), and provide an interesting observation.

Assume that the channel is approximated with an FIR model of length $n h$. Also assume that $C=D=1$ in the model (3). Then, the channel shortening solution with an FIR filter with length $n w$ can be written as

$$
\mathbf{w}_{o p t}^{H}=\tilde{\mathbf{b}}_{o p t}^{H} \mathbf{R}_{x x} \mathbf{H}^{H}\left(\mathbf{H} \mathbf{R}_{x x} \mathbf{H}^{H}+\mathbf{R}_{n n}\right)^{-1}
$$

where $\mathbf{R}_{x x}$ is the $(n w+n h) \times(n w+n h)$ autocorrelation matrix of the input sequence and $\mathbf{R}_{n n}$ is the $n w \times n w$ autocorrelation matrix of the noise sequence. $\mathbf{H}$ is the $n w \times(n w+n h)$ 
convolution matrix defined by

$$
\mathbf{H}=\left[\begin{array}{ccccccc}
h_{0} & h_{1} & \cdots & h_{n h-1} & \cdots & 0 & 0 \\
0 & h_{0} & h_{1} & \cdots & h_{n h-1} & & 0 \\
\vdots & & \ddots & \ddots & \ddots & \ddots & \vdots \\
0 & 0 & \cdots & h_{0} & h_{1} & \cdots & h_{n h-1}
\end{array}\right]
$$

The vector $\tilde{\mathbf{b}}_{\text {opt }}=\left[0_{1 \times m} \mathbf{b}_{\text {opt }} 0_{1 \times n w^{\prime}+n h-n b-m}\right]^{T}$ is the $(n w+$ $n h) \times 1$ augmented target impulse response. The target impulse response vector $\mathbf{b}_{\text {opt }}$ under unit energy constraint can be found by minimizing

$$
J_{\min }=\mathbf{b}_{o p t}^{H} \mathbf{R} \mathbf{b}_{o p t}
$$

which is the eigenvector corresponding to the smallest eigenvalue of the matrix $\mathbf{R}$, where $\mathbf{R}$ is defined as

$$
\mathbf{R}=\Gamma^{H}\left(\mathbf{R}_{x x}-\mathbf{R}_{x y} \mathbf{R}_{y y}^{-1} \mathbf{R}_{y x}\right) \Gamma
$$

where $\mathbf{R}_{x y}$ is the $(n w+n h) \times n w$ crosscorrelation matrix of the input sequence and the shortening filter input, $\mathbf{R}_{y y}$ is the $n w \times n w$ autocorrelation matrix of the shortening filter input and $\Gamma=\left[\begin{array}{lll}\mathbf{0}_{n b \times m} & \mathbf{I}_{n b} & \mathbf{0}_{n b \times n w+n h-n b-m}\end{array}\right]^{T}$.

It is observed in the simulations that for minimum phase systems, as the length of the FIR filter increases, the optimum TIR solution in (23) approaches the solution obtained by the IIR channel shortening filter in (19). Similarly, the impulse response of the FIR shortening filter also approaches that of the IIR filter. Moreover, the term $\mathbf{R}_{x x} \mathbf{H}^{H}\left(\mathbf{H R}_{x x} \mathbf{H}^{H}+\mathbf{R}_{m n}\right)^{-1}$ in the solution for the FIR filter approaches the equivalent impulse response of $A N / \beta$ in the IIR filter based solution. The FIR channel shortening filter compensates the poles of the IIR filter (in fact corresponds to $\beta$ ) by placing zeros.

For a maximum phase system, since the IIR filter has to place its poles inside the unit circle ( $\beta$ is strictly stable), it cannot exactly cancel/compensate the maximum phase zeros of the channel, but places the filter poles to their conjugate inverses resulting in a whitening filter. The numerator polynomial on the IIR channel shortening filter, however, places appropriate zeros to further shorten the resulting channel.

\section{Simulations}

It is assumed that the channel is given in a pole-zero form. If this is not the case, without loss of generality the pole-zero equivalent of an IIR filter can be calculated, i.e. employing the method in [3].

Poles of a channel can easily be cancelled by the zeros of the shortening filter, therefore the issue of cancelling zeros of the channel is a more important issue. When a zero gets closer to the unit circle, the shortening filter needs to put a pole close to that position, which increases the length of the impulse response of the filter. In the simulations we examine this phenomenon by considering a channel having a conjugate pair of zeros and a conjugate pair of poles, and by fixing the location of the poles but placing the zeros at different positions inside the unit circle.

Two performance criteria will be considered. The first one is the compression ratio of the shortening filter, defined as the ratio of the energy of the signal inside the desired response window to that of the signal outside the window. The second criterion is the shortening signal to interference plus noise ratio (SINR), similar to the first criterion but noise amplification is also included to the interference term.

For a fair comparison, the implementation complexity (i.e. number of coefficients) of both IIR and FIR filters are kept the same, that is, if the IIR filter has $p$ poles and $q$ zeros, the compared FIR filter is designed with $p+q+1$ taps.

Figure 2-4 depict the comparison of the simulation results of the proposed IIR design with the FIR MMSE-UEC approach. The channel is chosen such that the poled are fixed at $0.8 e^{j \pm \pi / 4}$, but the position of zeros $r e^{j \pm \theta}$ are allowed to change. We also fix the input ARMA model to $C=D=1$ and noise model to $M=1-0.3 q^{-1}, N=1+0.7 q^{-1}$.

In Figure 2, the time domain shortening performance of both approaches is demonstrated together with the original channel when the zeros are set to $0.95 e^{ \pm j \pi / 3}$, at $10 \mathrm{~dB}$ SNR at the shortening filter input. The channel is shortened to two taps $(n b=1)$, and the optimum delay turned out to be $m=0$. It has been found that $67 \%$ of the energy of the original channel is contained in the desired window which spans the first two samples. The FIR channel shortening filter can increase this ratio to $85.4 \%$ while the proposed IIR based scheme can achieve a compression of $99.6 \%$.

Figure 3 compares the SINR performance of the two methods by varying the radius $r$ of the zeros (when $\theta$ is fixed at $\pi / 3$ ) in Subfigure 3.a and by varying the angle $\theta$ (when $r$ is fixed at 0.95) in Subfigure 3.b. All parameters except the location of the poles are the same as those in Figure 2. It can be seen that the performance of both methods is very close when the radius $r \leq 0.4$. But as the zeros get closer to the unit circle the proposed method outperforms the FIR counterpart justifying the motivation of our study. Subfigure 3.b shows that the performance is very sensitive to angle but the performance of the IIR filter is either the same or better than that of the FIR filter.

Figure 4 provides the SINR comparison of IIR and FIR filters with respect to the signal-to-noise ratio (SNR) at the input of the filters. The zeros are located at $0.95 e^{j \pm \pi / 3}$. The IIR filter always outperforms the FIR filter, specially at high SNR region, as in this region the compression ratio plays a major rate in the SINR definition.

\section{CONCLUSIONS}

We proposed a novel design method for IIR channel shortening filter design under the MMSE criterion. The method involves a very general framework which includes ARMA data and noise processes and rational channel transfer function. Special cases such as FIR channel impulse response, white or colored noise models can easily be obtained from this general framework. We demonstrated that the proposed scheme outperforms an FIR channel shortening filter under most conditions, specially when the zeros of the channel get closer to the unit circle. Although the IIR filter appear to amplify the noise, its superior compression performance 


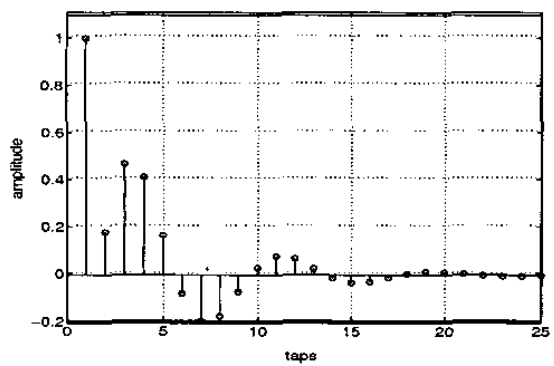

(a)

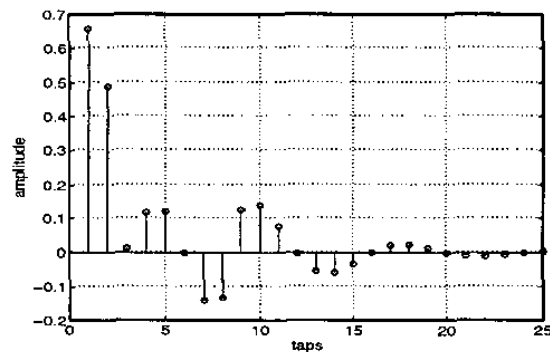

(b)

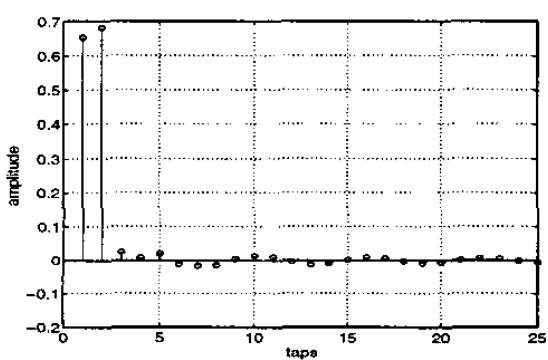

(c)

Fig. 2. Impulse response of (a) the original channel, (b) the equalized channel using a length eight FIR filter, (c) the equalized channel using the proposed IIR filter with four zeros and three poles.

compensates the noise amplification when SINR is considered as the merit of performance.

\section{REFERENCES}

[1] A. Ahlen, M. Sternad, "Optimal deconvolution based on polynomial methods", IEEE Transactions on Acoustics, Speech, and Signal Processing, vol.37, pp.217-226. Feb. 1989,

[2] A. Ahlen, M. Sternad, "Wiener filter design using polynomial equations", IEEE Transactions on Signal Processing, vol.39, pp.2387-2399, Nov. 1991.

[3] N. Al-Dhahir, A.H. Sayed, J.M. Cioffi, "Stable pole-zero modeling of long FIR filters with application to the MMSE-DFE", IEEE Transaction on Communications, vol.45, pp.508-513, May 1997,

[4] N. Al-Dhahir, J.M. Cioff, "Efficiently computed reduced-parameter input-aided MMSE equalizers for ML detection: A unified approach", IEEE Transactions on Information Theory, vol.42, pp.903-915, May 1996.

[5] N. Al-Dhahir, J.M. Cioffi, "A bandwidth-optimized reduced-complexity equalized multicarrier transceiver", IEEE Transactions on Communications, vol.45, pp.948-956, Aug. 1997.

[6] G. Arslan. B.L. Evans, S. Kiaei, "Optimum channel shortening for discrete multitone transceivers", IEEE ICASSP, vol.5, pp.2965-2968, June, 2000,

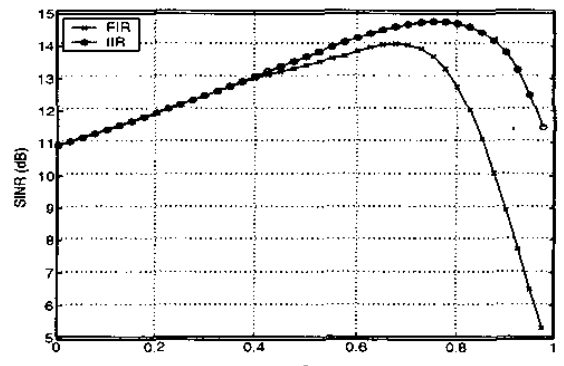

(a)

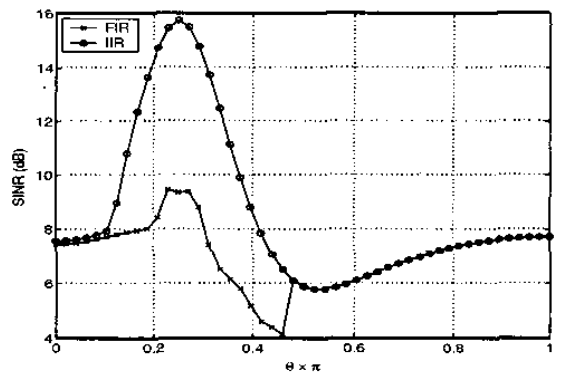

(b)

Fig. 3. SINR comparison of the proposed IIR filter with an FIR filter with respect to (a) the the radius of the zeros when the the angle is fixed at $\pm \pi / 3$, (b) the angle of the zeros when the radius is fixed at $r=0.95$. Both filters have the same implementation complexity.

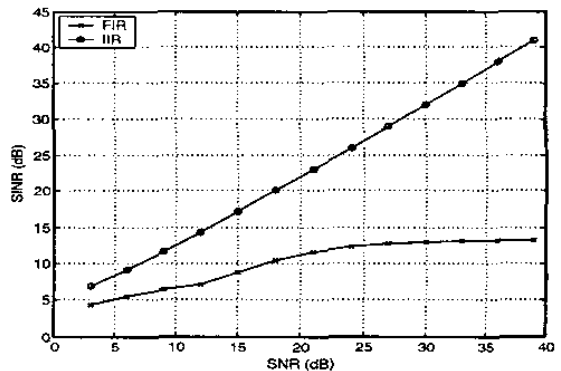

Fig. 4. SINR comparison of the proposed IIR filter with the FIR filter for various SNR at the channel output. Both filters have the same implementation complexity.

[7] M.V. Eyuboglu, S.U. Qureshi, "Reduced-state sequence estimation with set partitioning and decision feedback", IEEE Transactions on Communications, vol.36, pp.16-20, Jan. 1988 ,

[8] D. D. Falconer and F. R. Magee, "Adaptive channel memory truncation for maximum likelihood sequence estimation," Bell Systems Technical Journal, pp. 1541-1562, Nov. 1973,

[9] V. Kucera, Discrete Linear Control, The Polynomial Equation Approach, John Wiley \& Sons, 1979

[10] P.J.W. Melsa, R.C. Younce, C.E. Rohrs, "Impulse response shortening for discrete multitone transceivers", IEEE Transactions on Communications, vol.44, pp.1662-1672, Dec. 1996,

[11] C. Toker, S. Lambotharan, J.A. Chambers, B. Baykal, "Joint spatial and temporal channel shortening techniques for frequency selective fading MIMO channels", submitted to IEE Proceedings on Communications, June 2003 